\title{
Pervasive Accounting of Resource Consumption for Wireless Services with Adaptive QoS
}

\author{
Paolo Bellavista, Antonio Corradi, and Silvia Vecchi \\ Dip. Elettronica, Informatica e Sistemistica - University of Bologna \\ Viale Risorgimento, 2 - 40136 Bologna - ITALY \\ Phone: +39-051-2093001; Fax: +39-051-2093073 \\ \{pbellavista, acorradi, svecchi\}@deis.unibo.it
}

\begin{abstract}
Wireless communications and device miniaturization are enabling pervasive scenarios where the limited resource availability and the wide heterogeneity of access terminals make crucial to provide mechanisms and tools for the dynamic adaptation of Quality of Service (QoS). Particularly in case of very resource-consuming services QoS levels should be downscaled to fit the specific characteristics of the provisioning environment and of the current access device. In this context, a challenging aspect is to account clients not only for the generated network traffic, but also for the distributed resources involved in the dynamic QoS adaptation. The paper presents an accounting middleware solution that extends the traditional Internet where and when needed, depending on the mobility patterns of the wireless client devices, by introducing flexible mobile components for metering and charging. The middleware exploits the Mobile Agent (MA) technology to achieve dynamicity in metering/charging behavior, locality in the access to metering data, and to enable session-dependent accounting, even in case of temporary network partitioning.
\end{abstract}

\section{Introduction}

Telecommunication systems and the Internet are converging towards a pervasive integrated scenario with an enlarging set of very heterogeneous mobile devices, e.g., palm-sized computers, cellular phones and wireless pagers, that should access services anytime and anywhere. The ubiquitous availability of (either wired or wireless) networked computing environments identifies new service scenarios and requires new support solutions [1]. In particular, service provisioning to wireless portable devices should consider the strict limitations on their hardware/software characteristics, their wide heterogeneity, and the limited (and expensive) network resources typical of wireless environments.

The above aspects are particularly crucial when dealing with very resourcedemanding services, such as multimedia distribution. Some recent research work starts to recognize the need for middleware components that can dynamically downscale the Quality of Service (QoS) levels of Video on Demand (VoD) flows at the involved wireless access points over the fixed Internet infrastructure, in order to fit the characteristics of the specific provisioning environment, e.g., the screen resolu 
tion/size of current access devices, the expected average network bandwidth, and the user preferences $[2,3]$.

In this context, there is an emerging need for accounting solutions allowing the enforcement of QoS-aware charging and pricing strategies, which consider not only the service access duration time, but also the usage of all distributed resources involved in (the possibly adapted) service provisioning. Apart from billing purposes, rich and fine-grained accounting solutions could have the relevant side-effect of limiting the excessive resource demand by greedy users, thus reducing the risk of network/system congestion.

Accounting includes several management activities, from the metering of resource consumption, to the storing and processing of metering data. Metering should take into account several factors at different levels of abstraction, e.g., the type of accessed services, the requested QoS level, the generated network traffic, but also the processor time and the memory possibly consumed at intermediate nodes in case of dynamic QoS adaptation to client characteristics and preferences. Storing keeps metering data on stable storage, either in the locality where resource consumption has been measured or in a remote administration site with the additional transport and security issues. Processing elaborates the raw metering data to obtain concise indicators about service usage. When and how to perform processing may greatly vary depending on the accounting goals: in billing, metering data can be processed on/off-line to enable dynamic/static pricing strategies [4]; when dealing with QoS-aware services over best effort networks, processing can also be in charge of ascertaining the QoS level offered during service provisioning, both to perform QoS management operations and to charge users for the actually supplied QoS level [5].

The design and implementation of QoS-aware accounting systems in mobility scenarios has to face additional challenging issues. Mobility-enabled accounting requires location tracking while clients roam in global environments and coordinating remote resources in different networks, possibly with heterogeneous metering mechanisms. In addition, accounting systems should be capable of organizing charging strategies that may depend on mutual agreements between different service providers and network operators, and on previous user actions in different network localities. Moreover, mobility-enabled accounting should operate independently of possible temporary disconnection and network partitioning.

As already sketched above, the hardware/software limits of several categories of portable devices are pushing towards dynamic infrastructures to support their network connectivity, service access, and QoS differentiation. We claim that the same infrastructures should provide accounting functions to charge access devices for the resources actually consumed in any network locality willing to offer mobile accessibility. For instance, let us consider tourists equipped with Wi-Fi Personal Digital Assistants (PDAs) who like to access information about the historical buildings, monuments, restaurants, and shops in the downtown area where they are. If the area provides IEEE 802.11 service access points, the PDAs can potentially access the very detailed multimedia tourist information which is often already available in Web servers over the fixed Internet; to this purpose, however, this information usually has to be downscaled to fit the specific bandwidth/visualization capabilities of the PDAs. It is necessary to dynamically deploy novel support functions for content adaptation on the involved wireless access points; this infrastructure should monitor and charge tourists also for the resources consumed in the process of dynamically downscaling the service contents. 
The paper specifically focuses on solutions for the accounting of wireless devices in mobility contexts, i.e., pervasive accounting. We claim that pervasive accounting requires a dynamic and extensible support infrastructure, capable of evolving during service provisioning depending on client mobility. The accounting infrastructure should meter, register and charge resource consumption locally where mobile users/devices move to, without requiring continuous connectivity with remote and centralized accounting managers. The paper discusses and motivates the design of pervasive accounting middleware based on the Mobile Agent (MA) technology, to deploy the support infrastructure only when and where needed at service provisioning time. MAs can install new metering and charging behavior dynamically, can maximize locality in the access to metering data, and can enable accounting even in case of temporary network partitioning.

Along these guidelines, we have designed and implemented a pervasive accounting service on top of the Secure and Open Mobile Agent (SOMA) platform ${ }^{1}$. On the basis of a support layer for the portable metering of resource consumption, the pervasive accounting service is organized in terms of accounting MAs that follow client movements in the different wireless network localities. The co-locality of accounting MAs and their mobile users enable local metering, storing and charging depending on the actual consumption of system/application-level resources at service provisioning time. The pervasive accounting service presented in this paper integrates with our previous research work in multimedia QoS tailoring/adaptation over best-effort networks [6].

The paper is structured as follows. Section 2 presents the running case study of a tourist guide service with wireless accessibility and QoS dynamic adaptation of the provided VoD flows: the Downtown Visitor Assistant (DVA). The DVA scenario points out the requirements for pervasive accounting solutions and makes evident the need for the adoption of highly dynamic implementation technologies, such as MAs, as discussed in Section 3. Section 4 describes the layered architecture of the pervasive accounting service, while Section 5 and 6, respectively, give some implementation insights and show the accounting components at work during DVA provisioning. Related work, conclusive remarks and directions of current research end the paper.

\section{The Downtown Visitor Assistant Case Study}

The provisioning of the DVA service to wireless portable devices is a useful scenario to outline the issues related to QoS-aware pervasive accounting. In DVA, tourists are expected to use a standard Web browser running on their PDA while they roam in the city downtown. The DVA service is made available by Service Access Points (SAPs) offering Wireless Local Area Network (WLAN) connectivity; each SAP covers a single district of the downtown area.

The DVA service may be provided to wireless terminals as a set of service units, where a service unit is a set of Web pages (and included multimedia presentations) with logically correlated tourist information. For instance, a service unit may be the "virtual visit of the cathedral", which consists of a start HTML page and of a set of video/audio files; another one may be the "cinema and theater program", which is

1 Additional information about SOMA and its downloadable code are available at: http://lia.deis.unibo.it/Research/SOMA/ 
composed of a collection of HTML pages with embedded JPEG/GIF images. The above modeling and de-composition of service contents is also useful for the management of service costs, as illustrated in the following.

Any SAP plays the twofold role of both supporting wireless connectivity to the tourist information available on the wired Internet and adapting dynamically the service provisioning depending on the user/access device. In this scenario, for each service unit, WLAN providers are interested in charging users for two main provisioning costs:

- Wireless connectivity costs. They are the costs associated to supporting wireless accessibility to DVA. Accounting these costs should consider the consumption of communication resources between the SAP and the wireless access device, and can be measured in terms of access duration time and generated traffic over the wireless network.

- Tailoring costs. They are the costs associated to the QoS adaptation operations performed by the SAP to make the service unit fit the specific access terminal. Accounting these costs requires metering the consumption of resources, such as CPU and memory, for dynamically downscaling service contents to adapt service results to the bandwidth/visualization capabilities of the access devices, e.g., to convert MPEG multimedia flows into a sequence of JPEG fixed images with minor resolution or to simply discard images of unsupported formats.

Tourists accessing DVA should have the possibility to provide personal preferences (user profiles) during the subscription phase; similarly, device profiles should be available to describe the characteristics of all the different supported access terminals. When a tourist enters a WLAN with her wireless device, user/device profiles should be exploited to define how to dynamically adapt the service content and to configure the user-specific charging strategy, i.e., by specifying which metering data are relevant and which are not.

In addition, when tourists request a service unit, the local wireless provider should send them an Estimate of Charge (EoC), i.e., an evaluation of the provisioning costs related to the delivery of the requested service unit, including the communication resources to be provided and the QoS adaptation to be applied. The EoC approximates the actually imposed charge and is statically calculated before service provisioning, and may consequently differ from the final actual charge. Only if the tourist accepts the EoC, the provider goes on with service provisioning.

\section{Mobile Agents for Pervasive Accounting}

The DVA scenario points out how the merging of mobility, limited devices, wireless communications and the Internet is going to change service provisioning and management. Traditional support solutions for fixed networks do not suit these novel dynamic environments where users, devices and even service components can change their location at runtime.

MAs are a suitable and effective technology to face the challenging issues raised by providing service accessibility to wireless portable devices, first of all because of MA mobility. MAs can follow the portable device movements to maintain co-locality with the clients they work for, or can dynamically move close to needed resources and service components to preserve operation locality. In addition, they can keep the 
reached execution state and restore it when continuing their execution at the destination host. This is crucial in pervasive accounting for tracking the service session state and the resource consumption history of portable devices connecting to/disconnecting from different wireless network localities.

Pervasive accounting stresses dynamicity, as the possibility of modifying and extending the support infrastructure by installing/discarding the needed metering, pricing and charging behavior, in response to runtime evolving requirements. Dynamic distribution/modification of code and dynamic resource binding are very similar in case of both MAs and the QoS-aware support of portable device accessibility [2].

Service provisioning and accounting in the mobility-enabled Internet call for visibility of the location of client users/devices. Location awareness is crucial to adapt services to the currently available local resources and to enable accounting strategies depending on local conditions and requirements. Location awareness is typical of the MA paradigm that propagates location visibility up to the application level, thus allowing high-level service management operations and QoS adaptation.

Moreover, the possible MA autonomy from clients simplifies service personalization. While following the device movements, MAs can facilitate the tailoring of services to the device profile characteristics and to the user personal preferences. Ad-hoc tailoring MAs can perform client-specific content adaptation at service provision time and should be accounted for their usage of execution resources. For instance, an MAbased infrastructure for service accessibility can follow the change of location of access devices by migrating correspondingly the QoS adaptation logic, even depending on the resource availability in the new network locality; the same infrastructure should account the clients for the local resource consumption related to runtime tailoring operations.

Accounting also calls for security, to authenticate mobile users/devices, to charge the service usage in a non-repudiable way and to grant secrecy/integrity in communications. The MA research faced and is facing the complex security challenges inherent to the technology to favor the MA adoption. As a side-effect, several state-of-the-art MA systems provide rich security solutions a pervasive accounting service can significantly benefit from [7, 8]. Finally, pervasive accounting needs interoperability to interact, meter and control the consumption of resources and service components available in the new, statically unknown, localities where the clients move at service provisioning time. To face similar problems of interaction with unknown resources, the MA research has promoted interoperable and standard interfaces, e.g., via compliance with CORBA and related standards, such as the OMG Mobile Agent Systems Interoperability Facility (MASIF), and the FIPA specifications, which can favor the openness and portability of MA-based implementations of pervasive accounting services [9].

\section{The Pervasive Accounting Service: Outline}

We have designed and implemented a pervasive accounting service realized in terms of a layered facility infrastructure that supports the provision-time accounting of the resources actually consumed by mobile users with wireless portable devices.

We already pointed out how pervasive accounting can benefit from the MA technology; our accounting solution operates on top of the SOMA platform to take advantage 
of the MA features. SOMA is a Java-based general-purpose middleware for the design, development and deployment of MA-based applications in global, open and untrusted environments [3]. Our accounting solution mainly exploits two SOMA facilities: the monitoring support and the portable service support. The monitoring support can inspect and extract data about the state of the resource engagement at any node hosting the execution of SOMA agents: each SOMA execution environment is equipped with a monitoring module tracing the allocation of different types of resources, at both the system level and the application one [10]. The portable service support consists of a mobile proxy-based infrastructure for service discovery, binding, dynamic QoS tailoring, and delivery of service contents to mobile wireless devices [2].

Our pervasive accounting service consists of three different components: the Metadata Service, the Accounting Coordination Service and the Charging Service.

The Metadata Service maintains profiles of registered users, supported access devices, available service components and available pricing policies. It implements a partitioned and partially replicated directory service specialized for profiles; any metadata directory component keeps local copies of profile information and coordinates with other peers to achieve global profile visibility.

The Accounting Coordination Service rules both service provisioning and resource usage metering. It configures and coordinates metering/tailoring functions driven by the enforced pervasive accounting strategy: according to the dynamically retrieved service/user/device profiles, it chooses the most suitable QoS adaptation components and commands the monitoring module by setting monitoring configuration parameters to trace the resource consumption also due to the QoS adaptation operations.

The Charging Service computes and proposes the EoC to the users during the service setup phase, and, most important, produces the charging reports to bill responsible users for their actual resource usage. The Charging Service locally processes monitoring data by taking into consideration the pricing profile information to apply to the specific service session, and compiles charging report for each wireless network locality visited during the service session.

After this brief sketch of the architecture of our pervasive accounting service, the following section gives implementation insights about the different accounting components and the SOMA facilities crucial for the accounting process.

\section{The Pervasive Accounting Service: Implementation Guidelines}

To fully understand the implementation of the pervasive accounting service, let us preliminary introduce the concept of locality abstractions. Any kind of interconnected system, from simple intranet LANs to the Internet, can be modeled in SOMA in terms of suitable locality abstractions. Any network node hosts one place for MA execution; several places can be grouped into domain abstractions, each one usually corresponding to one network locality. For instance, in the DVA scenario one SOMA domain typically models one LAN, together with its local IEEE 802.11 SAP and the corresponding WLAN cell. In each domain, a default place is in charge of interdomain routing functionality.

In the following, we first describe which metering parameters the monitoring module can provide and how. Then, the section details how the Metadata Service handles and 
exploits different types of profile metadata for accounting purposes, and presents the main implementation guidelines of the services for Accounting Coordination and Charging.

\subsection{Monitoring Module}

The Monitoring Module (MM) is a local middleware component available on any SOMA place. MM can inspect and make visible a wide set of monitoring indicators about resource consumption, both at the system level and at the application one. At the system level, MM gets information about the processes working on local resources and about their usage of the communication infrastructure. For any process, it can report the process identifier and name, the CPU usage (time and percentage, of both the process and the composing threads) and the allocated memory (both physical and virtual). Network metering data include, for any process, the total number of sent/received UDP packets, of sent/received TCP segments, of TCP connections, and of TCP/UDP packets received with errors. At the application level, MM can collect information about all service components accessed from within the Java execution environment. For any active Java thread, it can detect any invocation of a dynamically definable set of methods and any object allocation/deallocation operation.

To overcome the transparency imposed by the Java Virtual Machine (JVM), MM exploits extensions of the Java technology: the JVM Profiler Interface (JVMPI) [11] and the Java Native Interface (JNI) [12]. In addition, MM integrates with external standard monitoring entities, i.e., Simple Network Management Protocol (SNMP) agents [13]. JVMPI makes possible to instrument dynamically the JVM for debugging and monitoring purposes, and MM exploits it to collect, filter and analyze applicationlevel events produced by Java applications. At the kernel level, MM collects systemdependent monitoring data, by interrogating SNMP agents that export local monitoring data via their standard Management Information Base (MIB). To enable also the monitoring of hosts without any SNMP agent in execution, MM exploits JNI to integrate with platform-dependent monitoring mechanisms, which we have currently implemented for the Windows NT, Solaris, and Linux platforms.

MM processes raw metering data to quantify concisely the resource consumption and the service usage. For instance, the network-related information is used to determine the overall network traffic generated by one user during her service session. The CPU usage and the memory allocation on the SAP currently providing connectivity to the wireless client can represent a rough estimate of the computational costs associated with dynamic QoS downscaling, e.g., when converting a VoD flow included in a DVA service unit from the AVI/WAV formats to the TealMovie proprietary one.

Let us point out that MM can be dynamically tuned by choosing both the type of relevant resources to monitor and the accuracy/frequency of the monitoring probing. This permits to reduce significantly the overhead of the metering functions. For additional details about the monitoring implementation and performance, see [10]. In addition, MM is in charge of locally maintaining the metering data in stable storage supports; these data are then collected and processed by the specialized charging MAs presented in Section 5.4. 


\subsection{Metadata Service}

The pervasive accounting service adopts high-level metadata, provided by the Metadata Service (MS), to describe the entities involved in service provisioning and the corresponding pricing strategies depending on actually consumed resources. MS exploits four types of profiles: user, device, service, and pricing profiles.

User profiles are generated in the user subscription phase and include information about users and their service preferences: personal data, security-related information (digital identity certificate), credit card number, subscribed services, and class of subscription (gold, silver, and bronze) to the WLAN provider.

Device profiles describe the hardware capabilities (type of processor, quantity of memory, graphic resolution, ...) and software characteristic (operating system, JVM version, supported graphic formats, ...) of the registered wireless access devices. User/device profiles are represented according to the W3C Composite Capabilities/Preference Profile (CC/PP) specification. Fig. 1 shows an example of device profile.

Service profiles describe the service units composing a service, in terms of operations and input/output type definitions. Each service unit implies a sequence of operations (getCathedralVisitStart, getCathedralHistoryVideo, ...) consisting of a set of requests/replies messages with specified types and formats (HTML, MPEG-1, ...); these operations define the service interface. The service profile describes this interface and plays a crucial role to evaluate the costs of the requested service unit, depending also on applicable pricing/device profiles, and to determine dynamically which kind of adaptation operations are needed, accordingly to the proper device profile. Service profiles are expressed in the Web Services Description Language (WSDL). Figure 1 shows an example of service profile in the case of the virtual visit of the cathedral.

Pricing profiles define the price for any resource consumed during service provisioning and are the basis to enforce charging policies and to determine final user bills. According to the DVA pricing strategy sketched in Section 2, our solution accounts users for resource consumption in terms of connection and dynamic QoS adaptation operations. Two pricing profiles are exploited: the connection pricing, and the adaptation pricing, each of them specifying the cost per unit of the corresponding accountable resources, i.e., access duration time and bandwidth occupation for the connection, CPU and memory for the adaptation. For instance, access duration and CPU are priced in $€ / \mathrm{s}$. Costs can vary with the time of the day and with the user class of subscription. The pricing profiles are expressed according to the Resource Description Framework (RDF) format. CC/PP, WSDL and RDF specifications can be found in the W3C web site [14].

We implemented a very simple pricing/tariffing strategy just to verify the feasibility of our approach. However, we believe that this accounting approach, which exploits profiles to describe how to price the service and performs distributed processing of monitoring data to charge users at provisioning time, is suitable for enforcing also more sophisticated and dynamic pricing policies. 


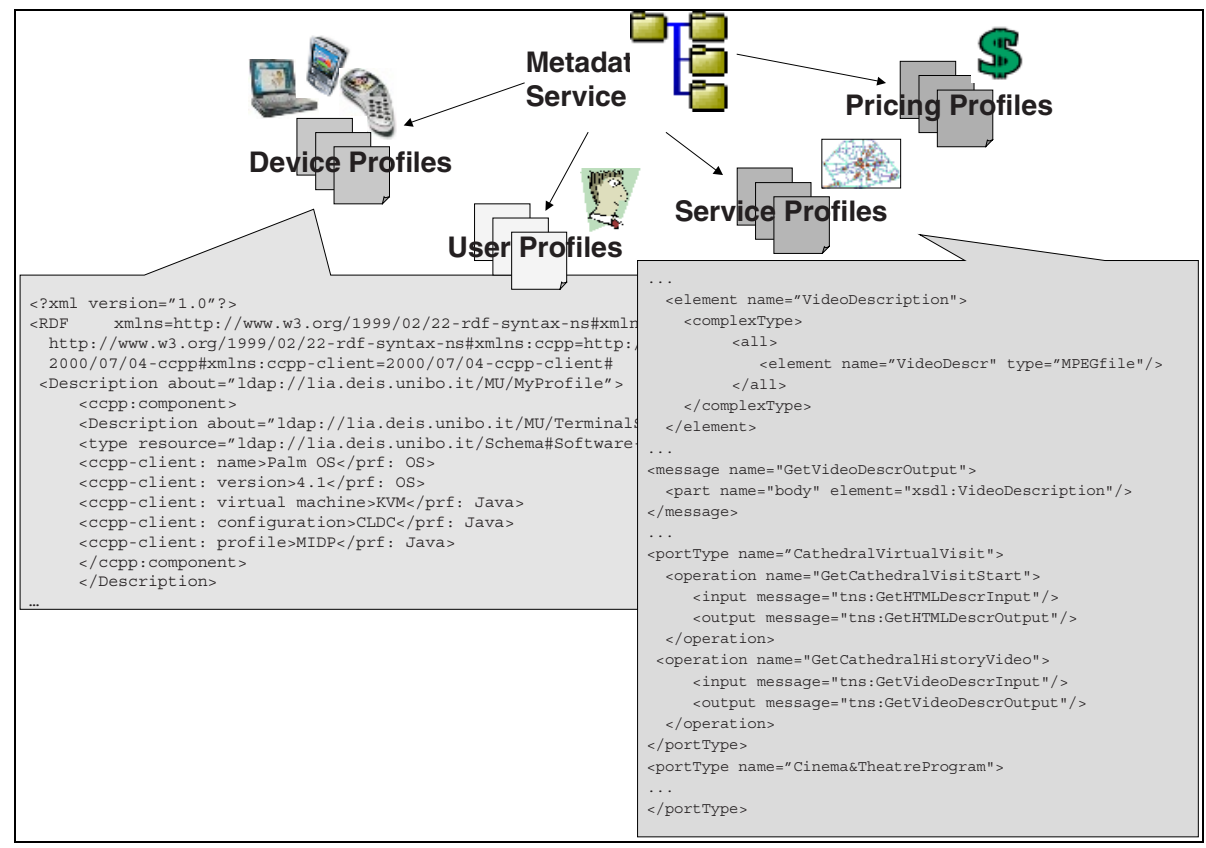

Fig. 1. Examples of device and service profiles

\subsection{Accounting Coordination Service}

The Accounting Coordination Service exploits the decentralized monitoring modules and the Metadata Service profiles to determine how, where and when to perform QoS-aware accounting management. The service is implemented in terms of support components, i.e., the Coordination Mobile Agents (CoMAs), which automatically and dynamically distribute to the involved wireless SAPs.

CoMAs are in charge of configuring the service downscaling and of specializing the resource metering according to the applicable profiles. In particular, the user, device and service profiles drive the decisions about the service management operations necessary for the dynamic QoS adaptation of service contents, while service and pricing profiles impact on the choice of the accountable resources to monitor. CoMAs are organized hierarchically: the CoMA manager decides the adaptation/metering operations to perform in accordance with profile metadata and delegates these actions to more specialized CoMAs, the Tailoring MAs (TMAs) and the Monitoring MAs (MMAs), operating locally to the interested SAPs.

TMAs can carry out different QoS adaptation operations by exploiting local basic filtering functions provided by service adaptation components [2]. Examples of filtering include transcoding of the hypertext representation format (from XML to WML, from HTML to C-HTML, from HTML to HTML without image tags, ...), VoD/image format transcoding (from AVI/WAV to MPEG-1, from AVI/WAV to 
Divx, from AVI/WAV to the TealMovie proprietary format, from TIFF to JPEG/GIF, ...), and VoD/image resolution/size/color-depth decrease.

MMAs set their local monitoring modules to trace the CPU usage and the memory allocation of their local TMAs working for the specific downscale, and to measure the connection costs (service duration time and bandwidth consumption) from the SAP to the wireless client. MMAs exploit the metering functions available on any SOMA place involved in service provisioning. MMAs can also install new metering functions to extend the monitoring module, where and when needed, by exploiting the SOMA support for code mobility.

Any CoMA manager relates to one authenticated user and works as her care-of entity: it is instantiated at the first user access to the service, and obtains the user profile from the SOMA MS component in the domain. Then, the CoMA manager follows the user movements by carrying the user profile with itself. Since the same user can access different subscribed services in different moments and with different devices, only at the request of a new service unit the CoMA manager retrieves the applicable device/service/pricing profiles and determines which TMAs and MMAs are needed in the current access.

\subsection{Charging Service}

The Charging Service accomplishes two tasks. It exploits the user/service/device/pricing profiles to provide the EoC for the requested service unit, and uses the metering data stored by MMAs and the pricing profiles provided by the MS for the on-line processing of the global service cost report to bill the user with. The service is implemented in terms of Charging MAs (CMAs).

As the CoMA manager, also a CMA instance is dedicated to one authenticated user during a session and works as her care-of entity by following the user movements. When the user requests a service unit, the associated CMA is in charge of evaluating its cost and of proposing the corresponding EoC to the client. The EoC is calculated on the basis of two elements: the estimated adaptation costs necessary for downscaling the requested service unit depending on the applicable user/device/service profiles, and the estimated connection costs for the delivery of the adapted data in the requested service unit. For instance, the middleware chooses the proper tailoring operation in accordance with the device and service profiles, then estimates the cost for this operation and for the transmission of the adapted data on the basis of statistics about previous service sessions, e.g., the conversion cost from AVI to MPEG-1 format is $\mathrm{X} € /(\mathrm{s} * \mathrm{MB})$, the transmission cost of MPEG-1 stream with resolution $\mathrm{Y}$ is estimated as $\mathrm{Z} € /(\mathrm{s} * \mathrm{Y})$.

CMAs also process resource usage data at provision time according to the pricing strategy to compile charging reports. On the basis of the costs of accountable resources indicated in the pricing profiles, CMA works locally to elaborate the resource consumption information and to calculate the cost for the service usage in any visited SOMA domain. When the user leaves a domain, the CMA sends the charging report related to that locality to a central billing authority, which uses the CMA reports to compile the final bill for customer charging. 


\section{The Pervasive Accounting Service at Work in the DVA Scenario}

To clarify how the accounting components interoperate and coordinate in an actual service scenario, we present them at work in the DVA accounting. We model each district of the downtown, i.e., each WLAN, with a SOMA domain, where the IEEE 802.11b Service Access Point (SAP) is provided by a default place and the other tourist information servers in the district correspond to SOMA places, as depicted in Figure 2.

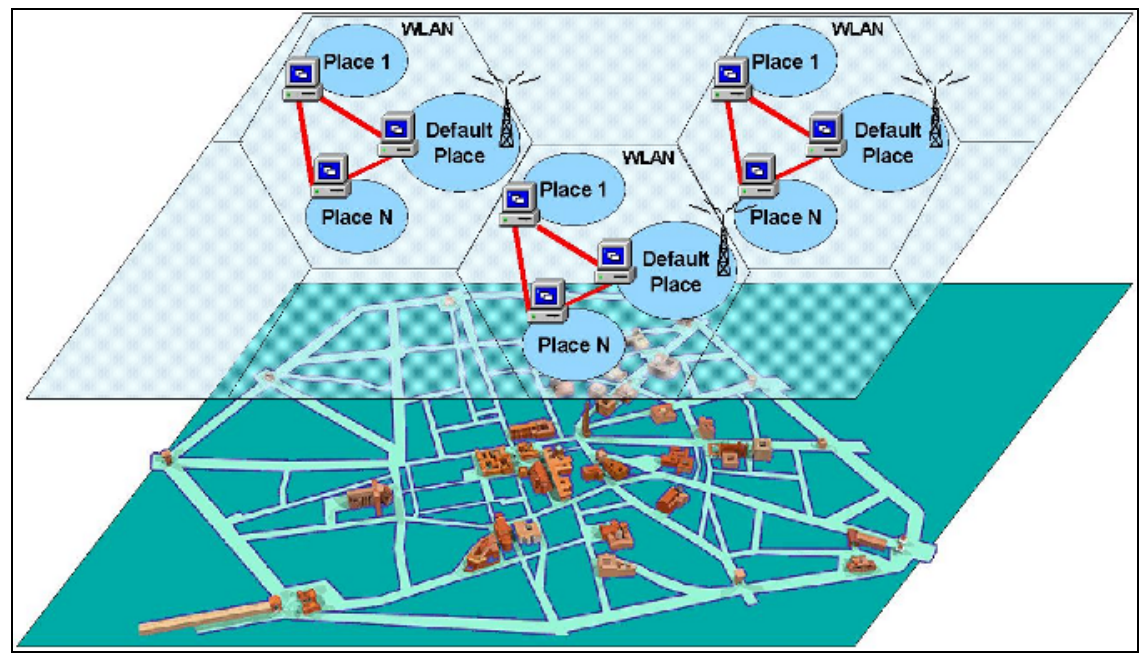

Fig. 2. Downtown district modeling in the DVA service.

Let us imagine a tourist $\mathrm{T}$ accessing the DVA service with an IEEE 802.11bcompliant PDA. When T enters a new domain, her entrance triggers a sequence of accounting management actions, as shown in Fig. 3. First, the system ascertains whether T already has a currently open accounting session; to this purpose, the system controls whether $\mathrm{T}$ has previously visited another close district/domain. If it is the first access to the service, the SOMA place currently providing wireless connectivity to the client asks MS for the user profile and then associates the tourist with newly instantiated CoMA manager and CMA. Otherwise, the middleware triggers the migration of the previously instantiated CoMA manager and CMA for that user from the last visited district/domain. In the worst case of network partitioning, if the already instantiated CoMA manager and CMA cannot reach the new domain, the system instantiates brand new CoMA manager and CMA for the user. To avoid CoMA and CMA proliferation, when CoMA managers and CMAs are inactive for a defined amount of time, they automatically terminate their execution.

When $\mathrm{T}$ requests a service unit, the CMA retrieves the involved user, service, device and pricing profiles (in the example, the profile of the user T, the DVA service profile, the Wi-Fi-enabled device profile, and, supposing T is a "gold" user, the "gold price" profile), and calculates and sends the $\mathrm{EoC}$ for the requested service unit, e.g. the virtual visit of the cathedral. If the tourist accept the EoC, the CoMA manager, by exploiting the involved service and device profiles, instantiates the suitable TMA and 
MMA. Since T accesses DVA from a PDA with limited screen size and graphic resolution, the required downscaling involves mainly the reduction of image size/resolution to fit the PDA-specific display capabilities. TMA performs these filtering/transcoding operations on the SOMA place with the SAP. MMA configures the local MM component on the same place to meter CPU usage and memory allocation of the selected TMA, and to measure bandwidth consumption to transmit the data composing the requested service unit. After this middleware reconfiguration, service provisioning and usage accounting can start, or continued in case of user roaming from a previously visited SOMA domain.

When T moves to another SOMA domain, the CMA charges locally the tourist service usage by processing data collected and stored in the domain by MM, according to customer category and pricing profiles: if $\mathrm{T}$ is a gold user and has visited the domain A from 10 a.m. until 12 a.m., CMA considers resource prices (time, bandwidth, CPU and memory) according to her gold class in the red (highest price) time. CMA creates a report for the visited domain with the cost for the local service usage and sends them to the central administration node, where reports are maintained in stable storage and finally processed to create a total usage-based bill for $\mathrm{T}$.

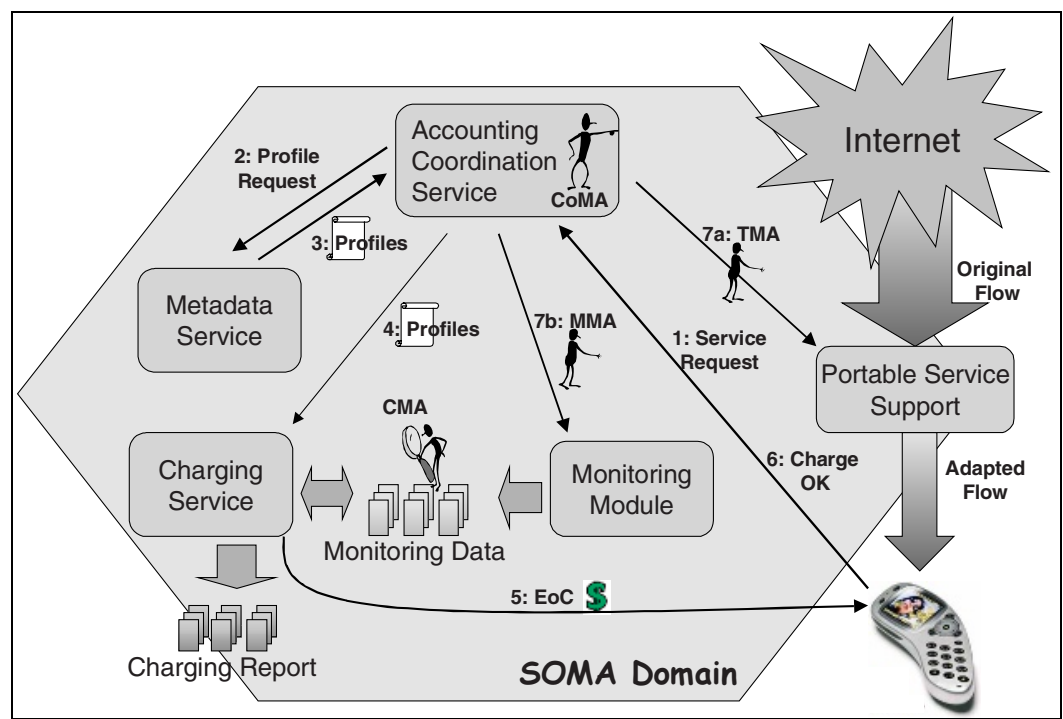

Fig. 3. Interactions of pervasive accounting components in a SOMA domain.

\section{Related Work}

Accounting is recognized as a relevant and challenging activity in the management of distributed resources, systems and services. IETF and IRTF have established working groups on Authentication Authorization Accounting (AAA) activities to deploy protocols and architectures to provide AAA services for the Internet. The most known and currently adopted solutions are Remote Authentication Dial In User Service (RADIUS) and Diameter $[15,16]$. These systems represent general-purpose manage- 
ment solutions mainly designed for traditional network infrastructures. They lack specific service management functions for pervasive accounting, and Diameter only faces the mobility issue by supporting mobile IP networks.

By considering the main accounting research activities on QoS and cost sharing aspects, on the one hand, the Market Managed Multiservice Internet project aims at enabling resource management on the basis of dynamically differentiated charging for different QoS levels. The essence of the market-managed approach is bringing the customer systems into the control loop through a mechanism of price tuning and new fare notification: support functions dynamically tune prices depending on client QoS demands; the proposed middleware infrastructure notifies users of the new prices and configures the accounting support accordingly, by exploiting a Java-based SNMPcompliant implementation [17]. On the other hand, the accounting working group of Next Generation Internet specifically focuses on the concepts of cost sharing and reverse charging, to enable an Internet Service Provider (ISP) to account final users for their actually received traffic, even when users are accessing the Internet via other collaborating ISPs [18].

By focusing on the accounting approaches that address also mobility issues, the Charging Accounting and Billing proposal, developed within the framework of the MOBIVAS project [19], is an integrated system for supporting advanced business models for service provisioning in $3 \mathrm{G}$ mobile systems. Its architecture allows flexible accounting, intended as the process of dynamic revenue sharing among Value Added Service Providers and home/visited network operators, depending on their mutual relationships and agreements. Another relevant project is MobyDick, which exploits the IPv6 mobility-enabled end-to-end architecture as the basis for its AAA charging infrastructure. In particular, MobyDick interfaces the DiffServ architecture with a dedicated Application-Specific Module that permits to control service access and to account roaming users/devices by adopting solution patterns similar to the mobile IP ones [20].

The above proposals confirm the need for increasing flexibility and dynamicity in pervasive accounting. They represent a significant evolution step in accounting solutions as they recognize the relevance of taking into consideration both user/device mobility and the provisioning of services with differentiated QoS levels. However, they follow the guideline of extending the functions of quite traditional management technologies and do not support the dynamic extensibility of the support infrastructure, the autonomy of accounting operations with regards to temporarily unreachable managers, and the metering of resources in visited networks considering consumed to dynamically adapt the provided QoS level. To the best of our knowledge, our pervasive accounting service is the first MA-based proposal specifically designed and implemented for highly dynamic pervasive scenarios where IEEE 802.11 SAPs provide and adapt service provisioning to $\mathrm{Wi}-\mathrm{Fi}$ access devices.

\section{Conclusions and Ongoing Work}

The Internet is likely to offer a pervasive service environment and this requires extending the fixed network infrastructure to support adapted service provisioning to mobile users and portable devices, when and where needed. In this context, effective accounting solutions should consider primarily the resources actually consumed dur- 
ing service provisioning; this is crucial to stimulate a fair resource sharing among users and to leverage the market of services with differentiated QoS levels. The paper shows how the accounting of portable client devices with wireless connectivity and limited hardware/software characteristics can significantly benefit from highly dynamic supports that exploit mobile code programming paradigms and, in particular, the MA technology.

The first encouraging results achieved in using the pervasive accounting service in the DVA case study are stimulating further research work to produce a more usable and complete accounting solution. Issues currently under investigation are nonrepudiation, micro-payments mechanisms, and mobile ad-hoc styles of interaction. From the security point of view, our accounting already exploits secure communications channels and encrypted storage for metering data. However, additional work is necessary to guarantee non-repudiability of resource usage and to establish differentiated trust levels between users and accounting components, so to minimize the security overhead when working in trusted environments. By focusing on payment aspects, a challenging issue is how to alleviate problems of fraud in mobile networks, by ensuring that all local bills are paid and by eliminating at the same time the need for inter-operator billing agreements. We are modifying the charging part of the accounting service to support also micro-payments mechanisms that can exploited by user-delegated CoMAs to operate local payments directly at service provisioning time [21]. Finally, we are our solution to account also services cooperatively provided by peer portable devices composing a proximity-based ad-hoc network, without exploiting a SAP infrastructure. This significantly changes the perspective and imposes to face additional challenges, such as how to measure resource consumption over very limited and heterogeneous access devices and where to store and process the accounting data.

Acknowledgement. This work is supported by the Italian MIUR (FIRB WEBMINDS project) and CNR (IS-MANET project).

\section{References}

1. G. G. Richard III, "Service Advertisement and Discovery: Enabling Universal Device Cooperation", IEEE Internet Computing, Vol. 4, No. 5, Sep.-Oct. 2000, pp. 18-26.

2. P. Bellavista, A. Corradi, C. Stefanelli, "The Ubiquitous Provisioning of Internet Services to Portable Devices", IEEE Pervasive Computing, Vol. 1, No. 3, Sept. 2002.

3. P. Bellavista, A. Corradi, C. Stefanelli, "An Integrated Management Environment for Network Resources and Services", IEEE Journal on Selected Areas in Communications, Vol. 18, No. 5, May 2000.

4. L. Da Silva, "Pricing for QoS-enabled Networks: a Survey", IEEE Communications Survey, Second Quarter 2000.

5. P. Bellavista, A. Corradi, S. Vecchi, "QoS-aware Accounting in Mobile Computing Scenarios", 11th Euromicro Conf. Parallel Distributed and Network-based Processing (PDP'03), Genoa, Italy, Feb. 5-7, 2003.

6. P. Bellavista, A. Corradi, "How to Support Internet-based Distribution of Video on Demand to Portable Devices", 7th IEEE Int. Symp. Computers and Communications (ISCC'02), Taormina, Italy, Jul. 1-4, 2002. 
7. A. R. Tripathi, N. M. Karnik, K. Manish, V. T. Ahmed, R. D. Singh, "Mobile Agent Programming in Ajanta", IEEE Int. Conf. Distributed Computing Systems (ICDCS 99), 1999.

8. N. Dulay, R. Montanari, C. Stefanelli, "Flexible Security Policies for Mobile Agent Systems", Microprocessors and Microsystems, Elsevier Science, Vol. 25, No. 2, Apr. 2001.

9. IKV++, Grasshopper 2: the Agent Platform, http://www.grasshopper.de/

10. P. Bellavista, A. Corradi, C. Stefanelli, "How to Monitor and Control Resource Usage in Mobile Agent Systems", 3rd IEEE Int. Symp. Distributed Objects and Applications (DOA'01), Sept. 2001.

11. Sun Microsystems - Java Virtual Machine Profiler Interface (JVMPI), http://java.sun.com/j2se/1.4/docs/guide/jvmpi

12. Sun Microsystems - Java Native Interface (JNI), http://java.sun.com/ j2se/1.4/docs/guide/jni/

13. J. D. Case, et al., "Simple Network Management Protocol (RFC 1157)", DDN Network Information Center, SRI International, May 1990.

14. W3C - The World Wide Web Consortium - http://www.w3.org/

15. C. Rigney, A. Rubens, W. Simpson, S. Willens, "Remote Authentication Dial In User Service (RADIUS)", IETF RFC 2138, Jan. 1997.

16. Pat R. Calhoun,Tony Johansson, Charles E. Perkins, "Diameter Mobile IP Application", http://search.ietf.org/internet-draft/draft-ietf-aaa-diameter-mobileip-14.txt

17. The Market Managed Multiservice Internet Project, http://www.m3i.org/index.html

18. A. Pras, B. van Beijnum, R. Sprenkels, R. Parhonyi, "Internet Accounting”, IEEE Communications, Vol.39, No. 5, May 2001.

19. M. Koutsopoulou, N. Alonistioti, E. Gazis, A. Kaloxylos, "Adaptive Charging Accounting and Billing system for the support of advanced business models for VAS provision in 3G systems", IEEE Int. Symp. Personal Indoor and Mobile Radio Communication (PIMRC'01), San Diego, USA, Oct. 2001.

20. H. Einsiedler, R. L. Aguiar, J. Jaehnert, K. Jonas, M. Liebsch, R. Schmitz, P. Pacyna, J. Gozdecki, Z. Papir, J. I. Moreno, I. Soto, "The Moby Dick Project: A Mobile Heterogeneous ALL-IP Architecture", Int. Conf. Advanced Technologies, Applications and Market Strategies for 3G (ATAMS'01), Cracow, Poland, Jun. 2001.

21. H. Tewari and C. O'Mahony, "Real-Time Payments for Mobile IP”, IEEE Communications Magazine, Feb. 2003. 